\title{
Inducible Mouse Models for Cancer Drug Target Validation
}

REVIEW

\author{
Joseph H. Jeong
}

Department of Developmental Biology and Genomics, College of Veterinary Medicine, Seoul National University and Korea Mouse Phenotyping Center, Seoul, Korea

\begin{abstract}
Genetically-engineered mouse (GEM) models have provided significant contributions to our understanding of cancer biology and developing anticancer therapeutic strategies. The development of GEM models that faithfully recapitulate histopathological and clinical features of human cancers is one of the most pressing needs to successfully conquer cancer. In particular, doxycycline-inducible transgenic mouse models allow us to regulate (induce or suppress) the expression of a specific gene of interest within a specific tissue in a temporal manner. Leveraging this mouse model system, we can determine whether the transgene expression is required for tumor maintenance, thereby validating the transgene product as a target for anticancer drug development (target validation study). In addition, there is always a risk of tumor recurrence with cancer therapy. By analyzing recurrent tumors derived from fully regressed tumors after turning off transgene expression in tumor-bearing mice, we can gain an insight into the molecular basis of how tumor cells escape from their dependence on the transgene (tumor recurrence study). Results from such studies will ultimately allow us to predict therapeutic responses in clinical settings and develop new therapeutic strategies against recurrent tumors. The aim of this review is to highlight the significance of doxycycline-inducible transgenic mouse models in studying target validation and tumor recurrence.
\end{abstract}

(J Cancer Prev 2016;21:243-248)

Key Words: Transgenic mouse, Drug targeting, Cancer, Doxycycline

\section{INTRODUCTION}

Recent explosive advances in high-throughput sequencing technologies have led to the identification of numerous somatic mutations in cancer genomes. ${ }^{1-6}$ However, only a few of them functionally contribute to tumorigenesis. Therefore, distinguishing 'driver' mutations that have a casual role in tumorigenesis from 'passenger' mutations, which have no effect on tumorigenesis, is critical for our understanding of tumorigenesis. Yet, if the driver mutations are not required for tumor maintenance, those gene products cannot serve as anticancer drug targets. ${ }^{7,8}$ Techniques used to assess whether the identified gene is associated with tumor maintenance in in vitro culture systems, include
siRNA-mediated gene expression knockdown or small chemical compound-mediated gene product activity inhibition. ${ }^{9}$ If these techniques abrogate gene product role in tumor cell growth, the gene may be required for tumor cell growth and therefore serves as a possible drug target for the inhibition of tumor growth. ${ }^{10-13}$

Several in vivo mouse systems have been used to verify these in vitro results. In particular, xenograft implantation systems have been extensively used to confirm in vitro data and to test the efficacies of small compounds for inhibiting tumor growth in mice. ${ }^{14-16}$ In these systems, either tumor cells or tumor tissues are implanted into the immunocompromised mice to reproduce tumor growth. These xenograft implantation systems have several advantages over genetically-engineered mouse (GEM)

Received November 2, 2016, Revised November 29, 2016, Accepted November 30, 2016

Correspondence to: Joseph H. Jeong

Department of Developmental Biology and Genomics, College of Veterinary Medicine, Seoul National University and Korea Mouse Phenotyping Center, Room 515, 1 Gwanak-ro, Gwanak-gu, Seoul 08826, Korea

Tel: +82-2-885-8389, Fax: +82-2-885-8397, E-mail: jeongjoseph@snu.ac.kr, ORCID: Joseph H. Jeong, http://orcid.org/0000-0001-6945-8157

Copyright (C) 2016 Korean Society of Cancer Prevention

(c) This is an Open Access article distributed under the terms of the Creative Commons Attribution Non-Commercial License (http://creativecommons.org/licenses/by-nc/4.0) which permits unrestricted non-commercial use, distribution, and reproduction in any medium, provided the original work is properly cited. 
models, such as being easy to prepare and use in a large cohort of mice with synchronized tumor growth. ${ }^{14}$ However, tumor growth in xenograft implantation systems frequently fail to faithfully recapitulate the genetics and histology of corresponding human cancers, partially due to the lack of microenvironmental factors, including stromal cell components and an immune system. ${ }^{14}$ In this regard, conventional transgenic mouse models in which the transgene is expressed under the control of a tissue-specific promoter/enhancer regulatory elements are a more physiologically relevant system for determining whether the transgene expression is sufficient for tumor development and progression. Unfortunately, there is no means to regulate (either induce or suppress) transgene expression in a temporal manner, making it impossible to determine whether the transgene expression is required for the maintenance of tumor phenotypes. To overcome this limitation, the inducible transgenic mouse model was developed. In this system, if turning off the expression of the specific gene in tumor-bearing transgenic mice shows that transgene expression is required for tumor maintenance, the suppression of transgene expression will likely lead to tumor regression, validating the transgene protein as a target for future anticancer drug development.

Given that tumor recurrence is always a concern in cancer therapy, understanding the molecular mechanisms underlying therapeutic resistance of tumor cells is critical for developing new therapeutic strategies against recurrent tumors. The majority of fully regressed tumors after turning off the expression of transgene by withdrawing doxycycline from the drinking water will reoccur in the original tumor site as transgene expression-independent. ${ }^{7,8,17}$ By analyzing these recurrent tumors, we can understand the molecular basis of how tumor cells escape from their dependence on doxycycline-induced transgene expression. ${ }^{17-19}$ Therefore, inducible mouse models enable us to extrapolate the collected in vivo data to predict therapeutic responses in clinical settings and develop new therapeutic strategies against recurrent tumors.

In this review, we will highlight the importance of inducible mouse models in studying target validation and tumor recurrence by citing several previously developed inducible mouse models as examples.

\section{THE TETRACYCLINE/DOXYCYCLINE (Tet)-INDUCIBLE TRANSGENIC MOUSE MODEL (THE Tet-ON SYSTEM)}

In the Tet-On system, the expression of the transgene is turned on (induced) by administering doxycycline via drinking water and turned off (suppressed) by withdrawing doxycycline from the drinking water. ${ }^{20.21}$ Two GEM models are required for this system. The first model harbors a gene of interest fused to a modified tetracycline response promoter element (TRE) that contains seven repeats of a 19-nucleotide tetracycline operator (tetO) sequence. ${ }^{20,21}$ The second model expresses an artificial transcription factor of tetracycline-inducible transactivator (rtTA) under the control of a tissue-specific promoter. To express the gene of interest in a specific tissue, these two mouse lines are crossed to generate compound mice harboring transgenes of both the gene of interest and rtTA. In the presence of doxycycline (adding doxycycline into the drinking water), doxycycline binding to rtTA enables the doxycycline-bound rtTA to stably bind to the TRE element, leading to the expression of the gene of interest in a specific tissue. ${ }^{20.21}$ In the absence of doxycycline (withdrawing doxycycline from the drinking water), doxycycline-free rtTA cannot bind to the TRE element, resulting in the suppression of the gene of interest.

\section{EXAMPLES OF INDUCIBLE MOUSE MODELS USED TO STUDY TARGET VALIDATION AND TUMOR RECURRENCE}

\section{Inducible mouse models for lung cancer}

Mutations in exons 18-21 of the human EGFR ( $h E G F R$ ) gene encoding the ATP-binding pocket of the receptor's tyrosine kinase domain are found in approximately $10 \%$ and $35 \%$ of patients with non-small cell lung cancer (NSCLC) in the US and in East Asia, respectively. ${ }^{22-25}$ In particular, an L858R substitution in exon 21 ( $h E G F R$ L858R) and an in-frame deletion in exon 19 ( $h E G F R$ DEL) are the two most common mutations. However, the identification of these recurrent mutations at high frequencies in clinical specimens does not necessarily mean that these mutations functionally contribute to the initiation and progression of NSCLC. Most importantly, for these mutations to serve as future therapeutic targets, these mutations should be required for the maintenance of NSCLC. Therefore, to determine whether these two mutations are associated with initiation, progression, and tumor maintenance of NSCLC, Dr. Wong's group developed inducible transgenic mouse models. ${ }^{26}$ Firstly, the group generated a transgenic mouse model in which either hEGFR L858R or hEGFR $D E L$ was fused TRE to generate two GEM mouse models: TRE-hEGFR L858R and TRE-hEGFR DEL. Secondly, these mice were crossed with Clara cell secretory protein promoter element-rtTA (CCSP-rtTA) mice to generate compound mice exp- 
ressing either $h E G F R L 858 R$ or $h E G F R D E L$ in lung type 2 alveolar cells in a doxycycline-inducible manner 26: TRE-hEGFR L858R/CCSP-rtTA and TRE-hEGFR DEL/CCSP-rtTA. Administration of doxycycline resulted in tumor development in murine lungs, serially precancerous lesions and bronchioloalveolar carcinoma within a few weeks, and invasive adenocarcinoma with more than four weeks after induction. ${ }^{26}$ In addition, withdrawal of doxycycline to suppress the expression of hEGFR L858R or $h E G F R$ $D E L$ in tumor-bearing compound mice resulted in complete tumor regression without recurrences. ${ }^{26}$ These results suggest that lung tumor cells are dependent on the expression of their respective transgenes for their proliferation and survival, validating these hEGFR mutant proteins as prospective drug targets for the treatment of lung cancers harboring these specific EGFRmutations. These findings were immediately applied in preclinical trials of pharmaceutical inhibitors specific to the EGFR mutants, such as gefitinib and erlotinib, to test their therapeutic efficacy against these inducible hEGFR mutant-driven mouse lung tumors. ${ }^{26}$ These tumor cells showed dramatic responses to the EGFR tyrosine kinase inhibitors, verifying that the EGFR mutants serve as drug targets for the treatment of human lung cancers harboring these specific EGFR mutations. In addition, these inducible hEGFR mutant mouse models were validated as valuable tools for efficacy studies of newly developed EGFR tyrosine kinase inhibitors.

However, in clinical settings, the majority of primary EGFR mutant non-small cell lung carcinomas that initially responded to EGFR tyrosine kinase inhibitors became resistant to the inhibitors. $^{27,28}$ Several genomic studies of recurrent patient tumor samples identified other genetic alterations, including the secondary mutation of EGFR T790M in the EGFR gene and mutations in the K-Ras gene..$^{23,29,30}$ Using another inducible mouse model that expresses a mutant $E G F R$ (EGFR TL) containing both $h E G F R$ L858R from the primary tumor and EGFR T790M from the recurrent tumor in murine lungs in the same manner above, it was shown that EGFR TL is oncogenic and essential for tumor maintenance. ${ }^{19}$ Given that an irreversible EGFR tyrosine kinase inhibitor, HKI-272, previously showed high efficacy against gefitinib-insensitive EGFR mutants, a therapeutic strategy combining HKI-272 with gefitinib was proposed to treat primary tumors driven by EGFR L858R and to prevent recurrent tumors driven by EGFR T790M. Preclinical trials of HKI-272 alone in EGFR $T L$-driven murine lung tumors showed suboptimal responses, consistent with unfavorable results from a phase I clinical trial of HKI-272 for the treatment of previously treated NSCLC patients. ${ }^{19}$ These studies suggest that results obtained from an inducible mouse model mirror clinical outcomes, validating inducible mouse models as promising tools for predicting clinical responses to cancer therapy.

\section{Inducible mouse models for breast cancer (HER/Neu and c-MYC)}

HER2/Neu is a member of the epidermal growth factor receptor family. HER/Neu protein overexpression and/or HER/Neu gene amplification were observed in approximately 20\% to 30\% primary human breast cancers, and were also associated with breast cancer progression and poor prognoses. ${ }^{31,32}$ To determine whether HER/Neu protein overexpression is associated with initiation, progression, and tumor maintenance of breast cancer, Dr. Chodosh's group developed inducible transgenic mouse models. ${ }^{33}$ Firstly, the group generated a transgenic mouse model ( TetO-NeuNT) in which a constitutively activate form of HER2/Neu with a substitution of valine for glutamic acid in the transmembrane domain (NeuNT) was fused to the minimal tet operator (TetO). Secondly, these mice were crossed with mouse mammary tumor virus promoter-reverse tetracycline transactivator ( $M M$ TV-rtTA) mice to express transgenes in the breast epithelia of the mammary ductal system. These MMTV-rtTA/TetO-NeuNT compound mice developed multiple invasive mammary carcinoma with pulmonary metastasis in the majority of tumor-bearing mice with doxycycline administration. ${ }^{33}$ After withdrawal of doxycycline, the tumors including pulmonary metastatic tumors, rapidly and fully regressed, suggesting that the activation of the HER2/Neu signaling pathway is required for tumor maintenance of both primary and metastatic tumors. ${ }^{18,33}$

Since tumor recurrence is concern of breast cancer progression, they investigated the molecular mechanisms underlying recurrent tumors derived from fully regressed tumors after turning off the transgene expression in this mouse model. ${ }^{18}$ This study demonstrated that Snail was upregulated in recurrent mammary tumors, and Snail overexpression is sufficient for inducing rapid tumor recurrence after suppressing the expression of the transgene NeuNT in a xenograft model using tumor cell lines derived from the primary mouse tumors. ${ }^{18}$ Therefore, Snailmay serve as a target for the treatment of recurrent breast cancer resulting from therapies against the HER2/Neu signaling pathway.

c-Myc gene amplification is also detected in up to $15 \%$ of human breast cancers, with a much higher frequency of approximately $50 \%$ in BRCA1-dysfunctional breast cancer. ${ }^{34}$ To investigate the functional contribution of $c-M y c$ overexpression in breast cancer development and progression, an inducible transgenic mouse model overexpressing c-MYC (MMTV-rtTA/TetO-c-Myc) 
was also generated by Dr. Chodosh's group. ${ }^{8}$ While $c-M Y C$ overexpression is sufficient for the development of mammary adenocarcinoma, approximately half of the primary tumors failed to regress after turning off the expression of the $c-M y c$ transgene. In addition, half of the fully regressed tumors recurred at the site of original primary tumor. ${ }^{8}$ Therefore, the majority of the initially c-Myc-driven tumors eventually lost their dependence on the c-MYC signaling pathway, suggesting that c-MYC may not be appropriate as a therapeutic target for the treatment of human breast cancers harboring $c-M y c$ gene amplification.

\section{An inducible mouse model for prostate cancer $\left(\mathrm{BRAF}^{\mathrm{E} 600}\right.$ )}

Many studies have reported the activation of the RAS/RAF/ MEK/ERK pathway in the majority of androgen-depletion independent (ADI) prostate cancers. ${ }^{35-37}$ However, counter-intuitively, activating mutations in the RAS/RAF/MEK/ERK pathway are infrequent in human prostate cancers. ${ }^{7,9}$ To study the role of the activation of the RAS/RAF/MEK/ERK pathway in prostate cancer initiation and progression in vivo, Dr. Chin's group generated an inducible mouse model (Tyr-rtTA/TetO-BRAF ${ }^{E 600}$ ), in which the expression of a potent activator of RAS/RAF/MEK/ERK signaling, $\mathrm{BRAF}^{\mathrm{E} 600}$, is targeted to the murine prostate epithelia by tyrosinase promoter/enhancer (Tyr). ${ }^{7}$ Although the tyrosinase promoter/enhancer has specific activity in melanocytes, it also showed activity in the prostate epithelia for unknown reasons. ${ }^{7}$ These mice developed invasive adenocarcinoma, and these tumors further progressed to indolent ADI lesions after castration. ${ }^{7}$ However, as in the case of the inducible $c-M y c$ transgenic mouse model, transgene $\mathrm{BRAF}^{\mathrm{E} 600}$-driven prostate tumors constantly grew even after turning off the transgene expression. ${ }^{7}$ Therefore, this study proposed that targeting $\mathrm{BRAF}^{\mathrm{E} 600}$ for the treatment of $\mathrm{ADI}$ prostate cancer does not have positive therapeutic potential.

\section{An inducible mouse model for pancreatic cancer (KRAS $^{\mathrm{G} 12 \mathrm{D})}$}

More than $95 \%$ of pancreatic ductal adenocarcinoma (PDAC) harbor mutations in the KRAS gene. ${ }^{38-40}$ The expression of $\mathrm{KRAS}^{\mathrm{G12D}}$ in murine pancreatic progenitor cells at a physiologically relevant level induced the full spectrum of pancreatic cancers from pancreatic intraepithelial neoplasias to invasive pancreatic cancer. ${ }^{41,42}$ These results suggest that the mutant $\mathrm{KRAS}^{\mathrm{G} 12 \mathrm{D}}$ is an oncogenic driver for the development of pancreatic cancer. To determine whether the mutant $\mathrm{KRAS}^{\mathrm{G} 12 \mathrm{D}}$ is required for tumor maintenance, Dr. Depinho's group generated an inducible mouse model wherein the mutant $\mathrm{KRAS}^{\mathrm{G} 12 \mathrm{D}}$ is expressed in murine pancreatic cells at a physiological level in a triple transgenic strain (tetO-Lox-Stop-Lox-KRAS ${ }^{\text {G12D }} /$ ROSA26Lox-Stop-Lox-rtTA-IRES-GFP/p48-Cre) in a doxycyline-inducible manner. In this mouse model, Cre expression in murine pancreatic cells eliminates the Lox-Stop-Lox cassette containing three repetitive transcriptional stop sequences from both tetOLox-Stop-Lox-KRAS ${ }^{\text {G12D }}$ and ROSA26-Lox-Stop-Lox-rtTA-IRES-GFP to express rtTA and GFP using the endogenous ROSA26 promoter and to generate tetO-KRAS ${ }^{\mathrm{G} 12 \mathrm{D}}{ }^{41}$ In the presence of doxycycline, the mutant $\mathrm{KRAS}^{\mathrm{G} 12 \mathrm{D}}$ is expressed in pancreatic cells of these triple transgenic mice. Leveraging this elaborate mouse model, they proved that mutant KRAS ${ }^{\mathrm{G} 12 \mathrm{D}}$ is required for the maintenance of mutant KRAS ${ }^{\mathrm{G} 12 \mathrm{D}}$-driven pancreatic tumors. ${ }^{41}$ To understand the molecular mechanisms of $\mathrm{KRAS}^{\mathrm{G} 12 \mathrm{D}}$-mediated pancreatic tumor maintenance in this mouse model, they analyzed changes in the transcriptome of mutant $\mathrm{KRAS}^{\mathrm{G} 12 \mathrm{D}}$-driven tumor samples at 24 hours after doxycyline withdrawal. They demonstrated that multiple metabolic pathways are down-regulated in mutant $\mathrm{KRAS}^{\mathrm{G} 12 \mathrm{D}}$-driven tumors upon the termination of transgene expression, suggesting that the mutant $\mathrm{KRAS}^{\mathrm{G} 12 \mathrm{D}}$ reprograms metabolism to enhance tumor growth. ${ }^{43}$ Therefore, this study proposes the possibility that mutant KRAS ${ }^{\mathrm{G} 12 \mathrm{D}}$ itself and associated metabolic pathways serve as drug targets for the treatment of mutant $\mathrm{KRAS}^{\mathrm{G} 12 \mathrm{D}}$-driven pancreatic cancers.

\section{CONCLUSION}

This short review article provides an overview of the significance of doxycycline-inducible transgenic mouse models in studying target validation and tumor recurrence by summarizing studies using inducible mouse models for lung cancer (TRE-hEGFR L858R/CCSP-rtTA, TRE-hEGFR DEL/CCSP-rtTA, and TRE-EGFR TL/CCSP-rtTA), inducible mouse models for breast cancer (MMTV-rtTA/TetO-NeuNTand MMTV-rtTA/TetO-c-Myc), an inducible mouse model for ADI prostate cancer (Tyr-rtTA TetO-BRAF $F^{E 609}$, and an inducible mouse model for PDAC (tetO-Lox-Stop-Lox-KRAS ${ }^{\mathrm{G} 12 \mathrm{D}} /$ ROSA26-Lox-Stop-Lox-rtTA-IRES-G FP/p48-Cre). Despite investing much effort and time, many researchers have failed to identify driver genes that functionally contribute to tumor development. When a driver gene is successfully identified, the driver gene may later be found not to be required for tumor maintenance. Thus, this driver gene product is not useful as a future drug target for the treatment of related cancers. These failures can be partially attributed to the limitations of in vitro and in vivo systems in studying target validation and tumor recurrence. ${ }^{16}$ For example, promising 
results regarding target validation through loss-of function study using either siRNA-mediated knockdown or small chemical inhibitor treatment with tumor cell lines are not frequently reproduced in in vivo systems. As mentioned above, conventional transgenic mouse models are limited for the study of target validation and tumor recurrence due to the inability to regulate transgene expression in a temporal manner. These limitations are overcome with an inducible transgenic mouse model.

Despite the significance of inducible mouse models in studying target validation and tumor recurrence, many were wary about the extensive use of doxycycline due to its detrimental effects on mitochondria. In particular, doxycycline impairs mitochondrial proteostasis and induces some physiological changes associated with energy metabolism in mice. ${ }^{44}$ In addition, doxycycline is a tetracycline antibiotic, so it can change the gut microbiome, ultimately affecting mice immune system and metabolism. ${ }^{45-47}$ These unwanted consequences might affect the tumorigenic potential of certain genes in this doxycycline-inducible mouse model. Therefore, the effects of doxycycline should be well controlled to study the tumorigenic potential of genes associated with immune responses and metabolic pathways in particular.

The ultimate goal of cancer research is to develop therapeutic strategies against primary tumors and recurrent tumors through the study of target validation and tumor recurrence. Therefore, inducible transgenic mouse models are indispensible tools for accomplishing this goal.

\section{CONFLICTS OF INTEREST}

No potential conflicts of interest were disclosed.

\section{REFERENCES}

1. Imielinski M, Berger AH, Hammerman PS, Hernandez B, Pugh TJ, Hodis E, et al. Mapping the hallmarks of lung adenocarcinoma with massively parallel sequencing. Cell 2012;150:1107-20.

2. Govindan R, Ding L, Griffith M, Subramanian J, Dees ND, Kanchi $\mathrm{KL}$, et al. Genomic landscape of non-small cell lung cancer in smokers and never-smokers. Cell 2012;150:1121-34.

3. Cancer Genome Atlas Research Network. Comprehensive genomic characterization of squamous cell lung cancers. Nature 2012;489:519-25.

4. Pietanza MC, Ladanyi M. Bringing the genomic landscape of small-cell lung cancer into focus. Nat Genet 2012;44:1074-5.

5. Cancer Genome Atlas Network. Comprehensive molecular portraits of human breast tumours. Nature 2012;490:61-70.

6. Burger PE, Xiong X, Coetzee S, Salm SN, Moscatelli D, Goto K, et al. Sca-1 expression identifies stem cells in the proximal region of prostatic ducts with high capacity to reconstitute prostatic tissue. Proc Natl Acad Sci U S A 2005;102:7180-5.

7. Jeong JH, Wang Z, Guimaraes AS, Ouyang X, Figueiredo JL, Ding $Z$, et al. BRAF activation initiates but does not maintain invasive prostate adenocarcinoma. PLoS One 2008;3:e3949.

8. Boxer RB, Jang JW, Sintasath L, Chodosh LA. Lack of sustained regression of c-MYC-induced mammary adenocarcinomas following brief or prolonged MYC inactivation. Cancer Cell 2004;6:577-86.

9. Jeong JH, Bhatia $\mathrm{A}$, Toth $\mathrm{Z}$, Oh $\mathrm{S}$, Inn $\mathrm{KS}$, Liao $\mathrm{CP}$, et al. TPL2/COT/MAP3K8 (TPL2) activation promotes androgen depletion-independent (ADI) prostate cancer growth. PLoS One 2011;6:e16205.

10. Pérez-Lorenzo R, Zheng B. Targeted inhibition of BRAF kinase: opportunities and challenges for therapeutics in melanoma. Biosci Rep 2012;32:25-33.

11. Karasarides M, Chiloeches A, Hayward R, Niculescu-Duvaz D, Scanlon I, Friedlos F, et al. B-RAF is a therapeutic target in melanoma. Oncogene 2004:23:6292-8.

12. Sumimoto H, Miyagishi M, Miyoshi H, Yamagata S, Shimizu A, Taira K, et al. Inhibition of growth and invasive ability of melanoma by inactivation of mutated BRAF with lentivirus-mediated RNA interference. Oncogene 2004;23:6031-9.

13. Wellbrock C, Ogilvie L, Hedley D, Karasarides M, Martin J, Niculescu-Duvaz D, et al. V599EB-RAF is an oncogene in melanocytes. Cancer Res 2004;64:2338-42.

14. Richmond A, Su Y. Mouse xenograft models vs GEM models for human cancer therapeutics. Dis Model Mech 2008;1:78-82.

15. Morton JJ, Bird G, Refaeli Y, Jimeno A. Humanized mouse xenograft models: narrowing the tumor-microenvironment gap. Cancer Res 2016;76:6153-8.

16. Holzapfel BM, Wagner F, Thibaudeau L, Levesque JP, Hutmacher DW. Concise review: humanized models of tumor immunology in the 21st century: convergence of cancer research and tissue engineering. Stem Cells 2015;33:1696-704.

17. Kwong LN, Costello JC, Liu H, Jiang S, Helms TL, Langsdorf AE, et al. Oncogenic NRAS signaling differentially regulates survival and proliferation in melanoma. Nat Med 2012;18:1503-10.

18. Moody SE, Perez D, Pan TC, Sarkisian CJ, Portocarrero CP, Sterner CJ, et al. The transcriptional repressor snail promotes mammary tumor recurrence. Cancer Cell 2005;8:197-209.

19. Li D, Shimamura T, Ji H, Chen L, Haringsma HJ, McNamara K, et al. Bronchial and peripheral murine lung carcinomas induced by T790M-L858R mutant EGFR respond to HKI-272 and rapamycin combination therapy. Cancer Cell 2007;12:81-93.

20. Jaisser $F$. Inducible gene expression and gene modification in transgenic mice. J Am Soc Nephrol 2000;11 Suppl 16:S95-100.

21. Saunders TL. Inducible transgenic mouse models. Methods Mol Biol 2011;693:103-15.

22. Lynch TJ, Bell DW, Sordella R, Gurubhagavatula S, Okimoto RA, Brannigan BW, et al. Activating mutations in the epidermal growth factor receptor underlying responsiveness of non-small-cell lung cancer to gefitinib. N Engl J Med 2004:350:2129-39.

23. Paez JG, Jänne PA, Lee JC, Tracy S, Greulich H, Gabriel S, et al. EGFR mutations in lung cancer: correlation with clinical response to gefitinib therapy. Science 2004;304:1497-500.

24. Pao W, Miller V, Zakowski M, Doherty J, Politi K, Sarkaria I, et al. EGF receptor gene mutations are common in lung cancers from "never smokers" and are associated with sensitivity of tumors to gefitinib and erlotinib. Proc Natl Acad Sci U S A 2004;101:13306-11.

25. Shi Y, Au JS, Thongprasert S, Srinivasan S, Tsai CM, Khoa MT, et 
al. A prospective, molecular epidemiology study of EGFR mutations in Asian patients with advanced non-small-cell lung cancer of adenocarcinoma histology (PIONEER). J Thorac Oncol 2014:9:154-62.

26. Ji H, Li D, Chen L, Shimamura T, Kobayashi S, McNamara K, et al. The impact of human EGFR kinase domain mutations on lung tumorigenesis and in vivo sensitivity to EGFR-targeted therapies. Cancer Cell 2006;9:485-95.

27. Herbst RS, Fukuoka M, Baselga J. Gefitinib: a novel targeted approach to treating cancer. Nat Rev Cancer 2004:4:956-65.

28. Muhsin M, Graham J, Kirkpatrick P. Gefitinib. Nat Rev Drug Discov 2003;2:515-6.

29. Pao W, Wang TY, Riely GJ, Miller VA, Pan Q, Ladanyi M, et al. KRAS mutations and primary resistance of lung adenocarcinomas to gefitinib or erlotinib. PLoS Med 2005;2:e17.

30. Pao W, Miller VA, Politi KA, Riely GJ, Somwar R, Zakowski MF, et al. Acquired resistance of lung adenocarcinomas to gefitinib or erlotinib is associated with a second mutation in the EGFR kinase domain. PLoS Med 2005;2:e73.

31. Berger MS, Locher GW, Saurer S, Gullick WJ, Waterfield MD, Groner B, et al. Correlation of c-erbB-2 gene amplification and protein expression in human breast carcinoma with nodal status and nuclear grading. Cancer Res 1988;48:1238-43.

32. Slamon DJ, Leyland-Jones B, Shak S, Fuchs H, Paton V, Bajamonde A, et al. Use of chemotherapy plus a monoclonal antibody against HER2 for metastatic breast cancer that overexpresses HER2. N Engl J Med 2001;344:783-92.

33. Moody SE, Sarkisian CJ, Hahn KT, Gunther EJ, Pickup S, Dugan $\mathrm{KD}$, et al. Conditional activation of Neu in the mammary epithelium of transgenic mice results in reversible pulmonary metastasis. Cancer Cell 2002;2:451-61.

34. Liao DJ, Dickson RB. c-Myc in breast cancer. Endocr Relat Cancer 2000;7:143-64.

35. Gioeli D, Mandell JW, Petroni GR, Frierson HF Jr, Weber MJ. Activation of mitogen-activated protein kinase associated with prostate cancer progression. Cancer Res 1999:59:279-84.

36. Thomas RK, Baker AC, Debiasi RM, Winckler W, Laframboise T, Lin WM, et al. High-throughput oncogene mutation profiling in human cancer. Nat Genet 2007:39:347-51.

37. Konishi N, Hiasa Y, Tsuzuki T, Tao M, Enomoto T, Miller GJ. Comparison of ras activation in prostate carcinoma in Japanese and American men. Prostate 1997;30:53-7.

38. Bardeesy N, DePinho RA. Pancreatic cancer biology and genetics. Nat Rev Cancer 2002;2:897-909.

39. Makohon-Moore A, Iacobuzio-Donahue CA. Pancreatic cancer biology and genetics from an evolutionary perspective. Nat Rev Cancer 2016;16:553-65.

40. Morris JP 4th, Wang SC, Hebrok M. KRAS, Hedgehog, Wnt and the twisted developmental biology of pancreatic ductal adenocarcinoma. Nat Rev Cancer 2010;10:683-95.

41. Aguirre AJ, Bardeesy N, Sinha M, Lopez L, Tuveson DA, Horner J, et al. Activated Kras and Ink4a/Arf deficiency cooperate to produce metastatic pancreatic ductal adenocarcinoma. Genes Dev 2003:17:3112-26.

42. Hingorani SR, Petricoin EF, Maitra A, Rajapakse V, King C, Jacobetz MA, et al. Preinvasive and invasive ductal pancreatic cancer and its early detection in the mouse. Cancer Cell 2003:4:437-50.

43. Ying H, Kimmelman AC, Lyssiotis CA, Hua S, Chu GC, Fletcher-Sananikone E, et al. Oncogenic Kras maintains pancreatic tumors through regulation of anabolic glucose metabolism. Cell 2012;149:656-70.

44. Moullan N, Mouchiroud L, Wang X, Ryu D, Williams EG, Mottis A, et al. Tetracyclines disturb mitochondrial function across eukaryotic models: a call for caution in biomedical research. Cell Rep 2015;10:1681-91.

45. Theriot CM, Koenigsknecht MJ, Carlson PE Jr, Hatton GE, Nelson AM, Li B, et al. Antibiotic-induced shifts in the mouse gut microbiome and metabolome increase susceptibility to Clostridium difficile infection. Nat Commun 2014:5:3114.

46. Langdon A, Crook N, Dantas G. The effects of antibiotics on the microbiome throughout development and alternative approaches for therapeutic modulation. Genome Med 2016;8:39.

47. Knight DJ, Girling KJ. Gut flora in health and disease. Lancet 2003:361:1831. 